\title{
Une innovation locale en sciences et techniques
}

essai pour une analyse de réseaux sociaux

Roads of installation of experimentation in Sciences

Xavière Lanéelle et Isabelle Harlé

\section{OpenEdition}

\section{Journals}

Édition électronique

URL : http://journals.openedition.org/educationdidactique/1133

DOI : 10.4000/educationdidactique.1133

ISSN : 2111-4838

\section{Éditeur}

Presses universitaires de Rennes

\section{Édition imprimée}

Date de publication : 30 décembre 2011

Pagination : 101-116

ISBN : 978-2-7535-1832-2

ISSN : 1956-3485

\section{Référence électronique}

Xavière Lanéelle et Isabelle Harlé, « Une innovation locale en sciences et techniques », Éducation et didactique [En ligne], 5-3 | 2011, mis en ligne le 30 décembre 2013, consulté le 08 décembre 2020. URL : http://journals.openedition.org/educationdidactique/1133; DOI : https://doi.org/10.4000/ educationdidactique.1133

Ce document a été généré automatiquement le 8 décembre 2020

Tous droits réservés 


\title{
Une innovation locale en sciences et techniques
}

essai pour une analyse de réseaux sociaux

Roads of installation of experimentation in Sciences

\author{
Xavière Lanéelle et Isabelle Harlé
}

1 L'innovation scolaire a longtemps été perçue avec méfiance par les politiques ministérielles; il faut attendre les années soixante-dix-quatre-vingt pour que l'institution accueille certaines "transgressions acceptables" à la suite de multiples rapports (les rapports Soubré et de Peretti en 1982, les rapports Legrand sur les collèges et Prost sur les lycées en 1983) (Cros, 2001, p. 48). Parallèlement, en 1985-1986 la gestion du système éducatif a été marquée par la décentralisation; ainsi l'État a transféré des compétences aux établissements et les a dotés pour ce faire d'un nouveau statut, celui d'établissement public local d'enseignement (ÉPLE). Depuis, le projet d'établissement a été rendu obligatoire en 1989, puis la Loi d'orientation et de programme pour l'avenir de l'école de 2005 s'est inscrite dans ce cadre en le prolongeant, l'innovation devient levier de changement. L'article 34 de la loi précise que «le projet d'école ou d'établissement peut prévoir la réalisation d'expérimentations, pour une durée maximum de cinq ans, portant sur l'enseignement des disciplines, l'interdisciplinarité, l'organisation pédagogique de la classe [...] ». Ces expérimentations contribuent à l'introduction de nouvelles pratiques pédagogiques, de dispositifs dérogatoires ${ }^{1}$ dans un lieu et un temps donnés. Une fois reconnues par le rectorat au titre de l'article 34 de la loi, ces innovations sont définies comme expérimentations.

2 Après avoir bénéficié de ce statut, si elles ont fait leurs preuves, les expérimentations sont intégrées de façon stable dans le projet d'établissement, instrument de l'autonomie reconnue aux ÉPLE, qui décline leur politique.

3 L'expérimentation en sciences, que nous avons choisie d'analyser ici, combine des enseignements de sciences (Sciences de la Vie et de la Terre [SVT], Sciences Physiques 
et Chimiques [SPC]) et des enseignements technologiques (Sciences de l'ingénieur [SI] : Électronique, Mécanique) dans un lycée.

4 Les innovations scolaires ont fait l'objet d'analyses diverses : historiques, l'innovation ayant longtemps été considérée comme une menace (Prost, 1983; Obin, 1998); systémiques, l'innovation-action étant toujours soumise à des effets de rétroaction de son environnement et potentiellement à l'entropie (Huberman, 1973; Havelock et Huberman, 1980); psychologiques sur le profil des innovateurs, confiants et prêts à s'engager dans une culture de l'organisation scolaire au service de l'apprenant (Gather Thurler, 1996 ; Giust-Desprairies, 1998), microsociales et/ou interactionnistes portant parfois une certaine attention aux réseaux sociaux (Wittorski, 1998; Gadéa, 1998). Enfin, les innovations ont également suscité de nombreuses monographies, principalement anglo-saxonnes ${ }^{2}$.

Parallèlement à l'expérimentation Hands-on de Léon Lederman, qui concerne les sciences et les techniques dans les écoles primaires des ghettos de Chicago et de New York dès les années quatre-vingts, suivie en France de l'« aventure de la main à la pâte ${ }^{3}$ » (Charpak, Léna et Quéré, 2005), initiée en 1995, et diffusée depuis au collège, on a assisté à une multiplication d'innovations dans l'enseignement des sciences et de la technologie comme par exemple l'enseignement intégré. La question du sens et de l'image des disciplines concernées par ces innovations interpelle, car les élèves manifesteraient ${ }^{4}$ peu d'intérêt pour les sciences, on pourrait en voir ensuite les effets dans l'érosion de l'orientation vers les filières scientifiques à l'université. Or, cette question constitue un enjeu politique: d'une part pour le système éducatif, c'est pourquoi respectivement l'inspection générale et des chercheurs s'en sont emparés (par exemple, Ourisson, 2002; Blais, Gaucher et Ottavi, 2008 pour ne citer que des philosophes; pour une synthèse des recherches sur la question voir Musset, 2009); d'autre part pour notre compétitivité, en effet sans scientifiques de haut niveau, peu de brevets. Cette question se pose à la France au sein de l'Union Européenne (HCSC, 2007) et plus globalement au sein des pays développés (OCDE, 2006). Il s'agirait alors de remanier les curricula européens (Rocard, et al., 2007), en s'appuyant sur les résultats de la recherche en didactique des sciences et en didactique de la technologie (Eurydice, 2006 ; Lebeaume, 2008). À cette fin, de nombreuses études ont été présentées en 2009 aux journées d'étude S-Team organisées pour le collège dans le cadre du Seventh Framework Progamme de l'UE, et aux journées d'étude INRP pour le lycée ${ }^{5}$. Elles avaient pour objet de questionner l'introduction dans l'enseignement secondaire de la démarche d'investigation en sciences et en technologie.

Ce rapide tour d'horizon serait incomplet si nous omettions les travaux de Françoise Cros (Cros, 1993, 1998, 2001, 2004 a et b) qui portent l'ambition d'une synthèse et d'un dépassement des approches sociologiques précédemment citées, grâce aux apports de la sociologie de l'acteur-réseau (ANT $\left.{ }^{6}\right)$. Cette approche offre l'opportunité bien saisir les enjeux et les modalités d'une innovation car :

- elle s'attache à analyser les associations d'actants aux enjeux parfois différents, toujours temporaires, qui se nouent autour de situations comme les expérimentations (cf. les expérimentations menées dans le laboratoire de Pasteur par Latour, 1984 ou la domestication de la coquille saint-jacques par Callon, 1986), et nous pensons que même si les pratiques d'enseignement dans le cadre des expérimentations art. 34 ont des résultats moins robustes que les sciences expérimentales, elles peuvent relever de la même analyse ; 
- elle remplace autant de causes que possible à l'action par des associations d'actants, aux configurations diverses, ce qui est précieux pour analyser des micro dynamiques locales qui s'inscrivent dans des dynamiques plus larges, liées à des associations d'acteurs institutionnels en amont, comme l'inspection générale, les législateurs.

7 Nous nous situons donc dans le prolongement des travaux de Françoise Cros qui a transposé la première la théorie de l'ANT dans le champ de l'innovation scolaire. De plus, comme Latour (2007), nous pensons aussi que « la tâche de définition et de mise en ordre du social doit être laissée aux acteurs eux-mêmes, au lieu d'être accaparée par l'enquêteur » (Latour s'appuie sur l'ethnométhodologie, p. 36). De plus, comme lui, nous avons élargi la notion d'acteur aux objets (matériel scolaire, espaces architecturaux) mais aussi aux choix législatifs (loi d'orientation). Enfin, la sociologie de l'acteur réseau exige, pour la scientificité de son propos, que le chercheur fasse « le compte rendu de la genèse hétérogène d'une construction" (p.132), ici l'expérimentation, lequel compte rendu permet de tracer un réseau, c'est-à-dire "une chaîne d'actions où chaque participant est traité à tous égards comme un médiateur [...] un récit, une description ou une proposition dans lesquels tous les acteurs font quelque chose [...] chaque maillon du texte peut devenir une bifurcation, un événement, ou l'origine d'une nouvelle traduction». (p.187-189). Lequel réseau permet de saisir quels sont tous les acteurs enrôlés ${ }^{7}$ (ou non) dans l'expérimentation, la dynamique qu'ils ont portée (ou ont entravée), la fragilité de la construction, puisqu'à chaque maillon une bifurcation peut se produire, au bénéfice ou aux dépens de l'expérimentation. Chaque maillon tient, au moins temporairement, grâce à la médiation des acteurs qui traduisent, c'est-à-dire qui mobilisent dans leurs discours des arguments adaptés, pour justifier et révéler le sens de leur action lorsqu'ils s'en font les porte-parole, susceptibles d'enrôler l'ensemble des actants autour d'un bien commun.

8 Françoise Cros a renouvelé les outils d'analyse des innovations scolaires (Cros, 2004b, p. 147-149). Une réflexion fondée sur ces outils permet-elle de dépister où et quand se situent les obstacles ou les ressources du chemin parcouru par les expérimentations? Nous nous proposons d'y répondre en conjuguant l'analyse de l'acteur-réseau à l'analyse des réseaux sociaux ${ }^{8}$, celle des chaînes relationnelles notamment (Grossetti, 2008).

9 À cette fin nous verrons les objectifs visés, les contenus, les impulsions, les ressources apportées par les acteurs et les tensions qui ont pu s'exercer à chaque maillon de la chaîne pour une expérimentation qui combine différents enseignements de sciences en LEGT. Nous avons choisi de présenter un seul cas, parmi les quatre que nous avons analysés jusqu'ici', compte tenu de son résultat, tel qu'il a été évalué par l'institution, c'est-à-dire négativement la première année ${ }^{10}$, plus positivement ensuite. La comparaison de ses caractéristiques aux différents moments : configuration du réseau, dynamique soutenue par les chaînes relationnelles, nous permet d'expliquer et d'objectiver l'échec de la première année et la relative amélioration de la seconde.

Pour étudier la mise en place de ces expérimentations, nous avons utilisé la méthode narrative de recueil des données, inaugurée par Michel Grossetti, sociologue des réseaux sociaux ${ }^{11}$, qui pour analyser l'émergence des innovations industrielles s'appuie sur des chaînes relationnelles. Cette méthode est compatible avec notre paradigme puisqu'elle permet de voir comment une configuration émerge au fil des récits qui reconstituent l'histoire des expérimentations. «Il s'agit de reconstruire des processus de montages de collaboration entre organisations, ou de construction d'une nouvelle 
organisation, et d'y faire apparaitre des situations de mobilisation de relations " (Grossetti, 2008, p. 96). Même si on peut faire un "arrêt sur image " à un moment donné. L'utilisation d'entretiens croisés permet d'éviter de se centrer sur un seul narrateur. On obtient ainsi des « narrations contrôlées".

11 Nous avons donc mené un entretien collectif (à l'automne 2007), et deux vagues d'entretiens semi-directifs croisés (une première vague en 2008, la seconde en 2009) auprès de l'ensemble des enseignants concernés, un entretien avec les deux chefs d'établissement qui se sont succédés, deux entretiens avec l'adjoint, un avec un IPR de $\mathrm{SPC}^{12}$, un entretien chaque année avec à chaque fois 2 élèves, pour mieux saisir la dynamique de l'expérimentation en en retraçant l'historique et le développement avec l'ensemble des actants enrôlés. Les entretiens ont été retranscrits et ont fait l'objet d'une analyse de contenu (Giglione et Matalon, 1978) centrée sur les objectifs affichés, les acteurs de l'impulsion, l'enchaînement des actions soutenu par les relations entre les acteurs lors de la mise en place de l'expérimentation, les ressources apportées, les tensions ou obstacles, l'éventuelle présence d'un leadership.

12 La méthode de Grossetti est intéressante puisqu'en croisant les récits des acteurs, elle débouche sur un compte rendu qui ne raconte pas une simple histoire mais met en évidence, par les contradictions entre les acteurs, les incertitudes, les tensions. Nous avons aussi analysé des sources documentaires (projet, bilans), suscité des « carnets de l'innovation" qui permettent aux enseignants de reconstituer l'historique par l'anamnèse et d'exprimer leur ressenti ${ }^{13}$; enfin nous avons mené une enquête par questionnaire auprès des élèves.

13 Après avoir présenté les résultats de l'observation de l'expérimentation et son évolution, nous montrerons, pour chaque année, en quoi une analyse en termes d'acteur-réseau conjuguée à une approche en termes de réseaux sociaux est heuristiquement féconde.

\section{Les débuts de l'option « science + » en classe de seconde au lycée Roberval}

L'expérimentation du lycée Roberval s'est construite autour d'un projet : élaborer des systèmes produisant de l'énergie renouvelable (éolienne, solaire, hydraulique, biomasse) mais elle ne donne pas entière satisfaction. Ainsi la MIVIP ${ }^{14}$ la juge confuse sous plusieurs aspects :

- l'expérimentation est affichée «option» alors que ce n'est qu'un atelier (il ne repose sur aucune obligation scolaire, aucun programme [il s'agit en fait de le dépasser]);

- l'expérimentation engagée ne correspond pas à l'expérimentation sur le papier (les mathématiques ont disparu);

- elle requiert des moyens suplémentaires, ce qui n'est pas dans l'esprit du moment dicté par la LOLF (même si elle est financée sur fonds propres);

- les objectifs sont ambigus. S'agit-il de renforcer l'intérêt pour les sciences ou de constituer une classe d'élite?

15 Elle ne donne ni satisfaction à l'inspection qui ne la suit pas, ni aux enseignants, ni à l'ensemble des élèves.

16 Nous avons d'abord pensé que l'expérimentation avait été impulsée par le proviseur et son adjoint, lequel nous a même "vendu», par un discours managérial, 
l'expérimentation, suscitée d'après lui par l'équipe de direction. Cependant, en considérant le "carnet de l'innovation " d'un enseignant, nous avons un doute sur cette impulsion puisqu'il écrit :

- le 29 septembre : « Nous avons fait la présentation de Sciences + aux élèves. Ceux-ci devaient en être informés par l'administration... qui ne l'a pas fait [...] On ne se sent quand même pas très soutenus par l'administration... »

- le 1er octobre «Je rencontre le proviseur-adjoint dans la salle des profs. Je lui parle de la réunion de présentation pour laquelle il devait prévenir les élèves. Il admet qu'il a oublié. »

En revanche, dans cette expérimentation, la loi d'orientation a joué un rôle, un inspecteur de SPC de passage ayant suggéré l'article 34 au proviseur. Notons que les IPR ne seront plus jamais ensuite mentionnés, ils ne se sont d'ailleurs pas déplacés. C'est pour répondre à l'injonction de la loi semble-t-il que le proviseur a été partie prenante. En effet, le texte de la loi vise avant tout les établissements; dès lors, ce sont leurs dirigeants qui sont évalués sur son application. Mais pourquoi alors ce désengagement? Cette absence de volonté d'assumer sa mission de leadership? Est-ce parce qu'il savait déjà à la rentrée que cette option était une fausse route compte tenu des objectifs non recevables par l'inspection?

L'expérimentation est donc orpheline de l'inspection et privée d'un leader institutionnel.

La seconde tension naît du fait que l'expérimentation est une refondation dont les enseignants ne se satisfont pas. La première mouture était, selon l'enseignant de SVT : "pluridisciplinaire avec les matières qui fonctionnaient un peu plus en parallèle, alors que maintenant on abonde beaucoup plus dans l'interdisciplinaire». Or, il est beaucoup plus difficile de coopérer que de juxtaposer comme dans la formule précédente, d'autant que cet enseignant de SVT a des relations tendues avec au moins un de ses collègues qui dénonce son "manque de conviction». De plus, les enseignants se sentent lésés : aucune ressource supplémentaire n'a circulé dans le réseau. Le volume horaire a été réduit (3 heures/semaine contre 4), et les professeurs craignent de "saborder" leur discipline. En effet, ils pensent que l'intérêt de l'administration tient plus à l'éventualité de la suppression du cloisonnement entre les matières (enseignement intégré), et donc du nombre de postes nécessaires au fonctionnement, que de l'intérêt didactique et pédagogique de l'option. En outre, ils ne bénéficient pas des heures de concertation demandées.

En entrant davantage dans la logique des acteurs, nous pouvons mieux comprendre les tensions autour de cette refondation. Le proviseur adjoint nous en donne l'occasion: " on avait notre petite pratique honteuse et on a voulu faire quelque chose de propre ", il s'agit donc pour la direction de l'établissement de placer l'option expérimentale dans un cadre légal : l'article 34. Néanmoins la légalité acquise ne suffit pas à l'administration, encore faut-il que l'atelier accroisse l'efficience de l'établissement, une des missions assignées or, c'est incertain. Le proviseur réduit donc le budget alloué, en gérant «des petits reliquats, vous savez on n'a pas beaucoup de marges en bout de course, avec cette marge on fait des choix ». Les intérêts sont donc contradictoires : les enseignants cherchent des ressources (heures rémunérées), le proviseur à gérer efficacement son budget. Le conflit reste néanmoins latent grâce aux heures tout de même attribuées.

De plus, le choix de cette mutation de l'expérimentation n'a pas contribué à enrôler l'ensemble des anciens participants puisqu'un enseignant de mathématiques s'est désengagé sous le prétexte de "problèmes d'emploi du temps", mais en fait parce qu' " ils 
n'arrivaient pas à voir comment ils peuvent faire des maths sans parler des maths!». L'enseignant de mathématiques nous confirmera qu'il était réticent à abandonner sa spécificité disciplinaire. Travailler ensemble certes, mais pas à n'importe quel prix. L'équipe l'a regretté mais s'en est accommodée.

Les élèves de Sciences + n'ont pas été tous enrôlés non plus, le chahut regretté par les enseignants de SI et le bris de matériel en sont les preuves tangibles. Les enseignants attribuent ce comportement à l'absence de notation de l'atelier. Pourtant l'enquête par questionnaire en fin d'année fait apparaître une certaine satisfaction (10 élèves sur 14 disent qu'ils ont été intéressés, les 4 autres sont sans doute ceux du groupe biomasse à l'origine du chahut).

Nous pouvons également noter l'absence de co-leadership chez les enseignants. Lors de l'entretien collectif, à chaque question, les quatre membres de l'équipe répondent successivement. On peut penser que tout le monde met un point d'honneur à traiter l'ensemble des collègues sur un pied d'égalité pour ménager l'harmonie du travail d'équipe qui vient d'être engagé. Mais peut-être s'observent-ils tout simplement? Défiance les uns des autres? Même l'enseignant de SVT, qui a fait partie de la première formule, n'est pas plus loquace. Alors que nous pensions que sa place allait être celle du leader, compte tenu de la compétence acquise par l'expérience, ce n'est pas le cas.

Autre obstacle : la confrontation avec la matière. L'élaboration de systèmes produisant une énergie renouvelable n'a rien de facile. Le vent, l'eau, les déchets sont des acteurs qui résistent ${ }^{15}$.

Quant à l'espace, il handicape les enseignants de SI, qui ont été "délocalisés » à un autre étage. Ils doivent ainsi soit transporter leur matériel d'un étage à l'autre ou monter et redescendre pour l'utiliser en cours de séance, ce qui occasionne perte de temps et d'énergie.

Des jugements de personne négatifs apparaîssent dans le dernier entretien: l'enseignant de SPC trouve les objets assez laids (systèmes producteurs d'électricité) et se dit déçu du travail réalisé sous la responsabilité du professeur de mécanique. Il juge que son collègue (qui n'a pas réalisé de maquettes) n'a pas le même souci pédagogique qu'il aurait - peut-être - eu. Celui-ci s'en dédouane : il est arrivé en cours d'année (lorsque le professeur de maths a fait défaut). Il regrette néanmoins que ces systèmes ne fonctionnent pas : la turbine de la mini-centrale hydraulique ne tourne pas assez vite pour générer du courant, «il eut fallu un kärscher » (pour la puissance du flux); le vent produit par un ventilateur était aussi insuffisant pour mouvoir l'éolienne ; quant au gaz de la décomposition des déchets de gazon, il a été inexistant, les élèves du groupe concerné ayant manifesté peu d'intérêt pour sa collecte. Seul le bateau solaire a fonctionné. Peut-être est-ce aussi pour se dédouaner qu'il critique ouvertement son homologue de SVT : «c'est vrai que quand il y a des choses à faire... c'est souvent, c'est pas lui qui fait les démarches!» Lequel enseignant n'a d'ailleurs pas renseigné de carnet. Ce dernier dit être "le seul à pratiquer une démarche d'investigation", et n'être pas " trop mécontent de ce qu'on avait fait, maintenant après, je sais que les collègues de SI, ça n'avait pas été ».

Le fait que ces jugements ne concernent pas des relations duales nous a interpellé : le professeur de SPC critique celui de SI, qui critique à son tour implicitement le proviseur-adjoint qui gère les salles ou qui critique l'enseignant de SVT, lequel soupçonne enfin dans certains de ses propos les deux enseignants de SI. Cette circularité montre qu'il n'y a pas eu de conflit ouvert; il nous a semblé qu'elle dénote 
simplement que chaque acteur cherche seulement à sauver la face, en faisant reporter sur un autre sa propre responsabilité.

Cette absence de cohérence dans le projet, de leadership et d'harmonie à ce stade de l'expérimentation est pénalisante. Aucune concertation véritable n'a eu lieu de la date de la rentrée à avril, en témoigne le "carnet de l'innovation" d'un enseignant: "Réunion sciences + avec le proviseur. Je n'aime pas les réunions pendant la récré, c'est trop court » et bientôt l'équipe changera de thème - le Rectorat accorde son autorisation pour trois ans - sans avoir fait de véritable bilan. La MIVIP a déploré que le rapport probablement aussi réalisé pendant une récréation - tienne en une page.

\section{De l'importance des enchaînements et des flux de ressources transitant par les réseaux}

L'expérimentation de Roberval ne présente pas les caractéristiques d'un succès, telles qu'elles sont importées par Françoise Cros dans son analyse des innovations scolaires (2004, p. 147-160).

30 Certains actants présents ne sont pas enrôlés : ils dressent des obstacles plutôt que d'apporter des ressources, ainsi en est-il d'une partie des élèves et de l'espace. Le réseau sociotechnique est étroit puisqu'il ne dépasse pas les limites de l'établissement. Le laboratoire, "un lieu ou un moment d'élaboration et de travail sur la problématisation de l'action ou de l'innovation autour de l'élaboration de concepts, de théorisation, de vérification, voire de régulation » n'apparaît pas : les enseignants ne se réunissent pas pour élaborer des préparations et problématiser leur enseignement. Les investissements de forme, " dispositif matériel permettant à l'action de se développer " n'en sont donc pas issus, le bilan d'une page réalisé le temps d'une récréation en étant la seule trace collective. Force est de constater aussi que la controverse sur l'objet pédagogique et didactique n'a pas eu lieu. Quant au bien commun, qui devrait apparaitre dans les objectifs, il est discutable.

31 Si nous prolongeons l'analyse à l'aide des travaux récents de Latour (2007, p. 187) c'està-dire en étudiant les maillons du réseau qui tiennent «au moins temporairement, grâce à la médiation des acteurs qui traduisent » et que nous examinons de manière dynamique ces maillons grâce aux chaînes relationnelles, nous pouvons voir comment la dynamique s'est grippée. Chaque maillon peut être l'occasion d'une bifurcation, or celles qui ont eu lieu ont éloigné l'expérimentation d'un chemin qui aurait pu être évalué positivement par l'ensemble des acteurs.

Prenons un maillon en exemple: au début de l'expérimentation les actants sont en place mais le proviseur-adjoint porte un jugement négatif sur le travail effectué dans le passé, témoignant ainsi d'un manque de reconnaissance du travail des enseignants. Dès lors ceux-ci diront "ils nous met des bâtons dans les roues". Une première bifurcation aurait pu avoir lieu, les enseignants auraient pu abandonner leur projet; mais une autre bifurcation aurait pu également se produire : avec un regard positif, une réserve exprimée de manière différente (nous pouvons imaginer que le proviseur ait lancé un défi en disant: "c'est intéressant, mais vous pouvez faire encore mieux»), les enseignants se seraient peut-être davantage impliqués, menant des investissements de forme au bénéfice de leur projet et fortifiant ainsi le maillon. La chaîne n'est pas 
automatiquement linéaire et orientée vers un progrès, elle peut être l'occasion de retours en arrière ou elle peut se briser.

À Roberval, la chaîne relationnelle est placée sous le sceau de tensions multiples, elles ne sont pas résolues avant de passer au maillon suivant, empêchant l'expérimentation de se réformer et d'avoir in fine une évaluation plus positive. L'expérimentation se met en place de manière confuse et insatisfaisante pour l'ensemble des acteurs concernés, qui dénoncent les rendez-vous manqués avec le proviseur, le manque d'implication des enseignants. De plus, aucun leader ni aucun traducteur n'accompagne l'expérimentation.

La chaîne relationnelle a des maillons si faibles que l'acteur-réseau fait finalement défaut.

Il nous semble par conséquent que les développements récents de l'ANT, conjugués au travail préalable qui avait été réalisé par Cros et à l'analyse des chaînes relationnelles permettent de mieux comprendre les processus à l'œuvre dans les expérimentations scolaires. Des caractéristiques comme la présence d'un laboratoire, de controverses, d'opérations de traduction sont des points de passage obligés (PPO), car une expérimentation, fragile par définition, «ne peut passer partout mais seulement en quelques endroits. Il suffit alors de concentrer les forces en ce point pour que la faiblesse se change en force » (Latour, 1984, p. 51). Mais la force des maillons, liée à des ressources suffisantes, facteurs de tensions résolues, est aussi un point de passage obligé.

\section{Essayer à nouveau}

L'expérimentation s'est donc poursuivie une deuxième année malgré le maigre bilan qui en avait été fait.

En 2008-2009, l'équipe, composée des mêmes membres, porte un regard réflexif sur son action: " on n'était pas forcément bien rodé. Là, on a plus cadré avec le vécu qu'on a eu l'an dernier ». L'organisation horaire reste la même. L'objectif est désormais de concurrencer un autre établissement aux sections européennes.

Le projet s'articule autour de l'astronomie dont c'est l'année mondiale, thème co-choisi par deux des enseignants qui se partagent le leadership désormais (l'enseignante de SPC et un enseignant de SI). Il s'agit, après avoir compris la configuration du système solaire, les mouvements relatifs des planètes entrent-elles (SVT) et le fonctionnement optique d'une lunette astronomique (SPC), de la reconstruire virtuellement sur ordinateur (fabrication des pièces et assemblage). Enfin, il s'agit de produire concrètement un dispositif électronique permettant d'orienter la lunette sur une planète en en programmant la date et l'heure (SI). Le professeur de SVT, désirant faire un «+ " par rapport à son programme ${ }^{16}$, entreprend aussi de promouvoir une démarche d'investigation autour de la question : « la vie peut-elle exister sans oxygène? »

Les enseignants, en l'absence de professeur de mathématiques, apportent les notions de géométrie, de calculs mais si "bah! nous on est capable de les faire", cela reste insatisfaisant.

Cette année, les enseignants s'investissent davantage : un des enseignants de SI nous dit avoir effectué 5 heures de préparation pour chaque heure de cours (il a démonté une lunette et effectué les dessins en 3D de toutes les pièces avant d'effectuer ses 
préparations de séances); ils ont organisé une soirée astronomique avec l'association locale et un voyage scolaire au Futuroscope qui participe à cette année mondiale. Même le professeur de SVT, sur lequel est toujours porté un regard négatif : «il ne répond même pas aux mails", «il n'a fait pas de suggestions ", est davantage enrôlé. D'une part, parce qu'il a organisé une évaluation du voyage scolaire. D'autre part, parce qu'il a été inventif pour faire du «+» tout en ayant une démarche d'investigation, démarche que les autres n'ont pas. Un professeur de SI dit : «on apporte des connaissances et puis après on les met en application». Ainsi, lorsqu'il simule le mouvement de la lunette par ordinateur, et qu'à partir de là les élèves doivent retrouver une loi entrée-sortie (la relation entre la vitesse du moteur et la vitesse de rotation de la lunette), il ne s'agit pas d'une démarche d'investigation mais d'une démarche expérimentale tout au plus.

Cependant les enseignants n'organisent toujours aucune réunion et échangent plutôt par courriel, ces élargissements témoignent donc d'un plus fort enrôlement, mais d'un laboratoire toujours peu présent.

Pour ce qui concerne les élèves, ils ont été moins intéressés par l'option cette année : 3 élèves sur 14 présents jugent l'option intéressante, 8 non et 4 sont sans opinion. En analysant plus finement leurs réticences, un malentendu marque l'année : 8 élèves reprochent la notation, et surtout le fait que la note ait compté dans la moyenne. En effet, les élèves étaient persuadés qu'il n'y aurait pas d'évaluation quand bien même ils en avaient été prévenus. Ils ont manifesté un fort mécontentement au conseil de classe soutenus par l'enseignant de mathématiques présent (celui qui s'est désengagé) qui en a profité pour prendre violemment parti contre l'option. Cependant plus tard, en entretien, des élèves admettent qu'après tout ils font d'autres options facultatives notées (par exemple le latin) et considèrent finalement normal d'être évalués, quel que soit l'enseignement pour lequel on est engagé, et que cela compte dans la moyenne.

Le proviseur a réagi face au conflit et a accordé un entretien d'une heure à l'ensemble de l'équipe, afin d'interroger le devenir de la notation et d'apaiser les tensions. C'est la première fois que la direction apporte directement des ressources (reconnaissance, apaisement, négociation). Pourtant, en cours d'année, le principal-adjoint de l'établissement ne fera pas de bilan intermédiaire, estimant que nous en sommes les évaluateurs. Or, la commande institutionnelle est de porter un regard sociologique mais pas d'évaluer ${ }^{17}$.

Les actants non-humains continuent de poser problème : l'installation d'un soleil de trois mètres de diamètre a défié l'équipe lors de la journée portes ouvertes. Cependant les enseignants ont cette fois gagné. L'espace n'a pas joué cette année contre l'expérimentation puisque l'enseignant de mécanique a pu disposer de la salle du rezde-chaussée pour ses activités informatiques pendant que son collègue de génie électrique utilisait celle du premier étage où étaient déjà installés le matériel et les logiciels dont il avait besoin.

La première année, les enseignants ont regretté l'absence de lien avec l'inspection qui pouvait les aider à "travailler en interdisciplinarité [...] que les élèves puissent faire le lien entre les différentes matières ». Seuls ils n'y arrivent pas, en témoigne l'un d'eux qui

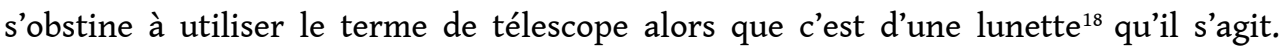
L'absence d'utilisation d'un vocabulaire commun est le symptôme d'un faible degré d'interdisciplinarité. Aujourd'hui l'inspection se sent concernée, elle dresse un bilan favorable à la poursuite de l'expérimentation et affiche qu'elle a pris conscience que les enseignants «se sont sentis livrés à eux-mêmes durant toutes ces années et sont demandeurs 
d'un accompagnement... qu'il convient que nous leur apportions ${ }^{19}$ ». Cet intérêt, quand bien même il n'aurait qu'un « effet Hawthorne ${ }^{20}$ » est porteur de ressources.

L'analyse en termes qualitatifs appuyée sur le schéma de Françoise Cros montre que le processus d'expérimentation a effectivement modifié par essais/erreurs la nature de l'expérimentation et $\mathrm{pu}$ dégager certains facteurs positifs d'évolution: réseau sociotechnique plus large, enrôlement de l'équipe et de son principal, mais elle ne permet pas de conclure à un bilan global totalement positif de cette expérimentation. Le bien commun, mieux défini, reste critiquable, les élèves sont moins satisfaits, l'année a été marquée par un conflit avec ces derniers et le professeur de mathématiques. Efficace pour opposer deux collèges Le Platane et le Marronnier aux résultats très contrastés, le modèle de Françoise Cros (2004b, p. 160) l'est moins lorsqu'il s'agit de mesurer une évolution complexe. L'analyse des réseaux permet-elle alors d'apporter un regard complémentaire qui objectiverait les résultats?

\section{Un essai d'objectivation par la quantification des ressources et des obstacles véhiculés par le réseau}

La sociologie des réseaux est plurielle. Une de ses branches s'attache aux chaines relationnelles, ce que nous avons fait jusqu'ici; une autre s'appuie sur la théorie des graphes. Elle mesure les réseaux complets ${ }^{21}$, ici celui de l'expérimentation qui réunit un ensemble d'actants, par le recours à des critères morphologiques (Mitchell, 1980). Ici, nous en utiliserons quelques-uns seulement :

- l'étendue (nombre d'actants impliqués). En effet, un réseau ouvert à des partenariats extérieurs est plus efficace, si c'est le cas alors l'étendue sera plus grande;

- la nature des liens (la relation peut apporter des ressources ou bien lever des obstacles). La nature n'est pas qualifiée arbitrairement par le chercheur ; elle est qualifiée par les acteurs eux-mêmes (cf. supra, Latour, 2007, 36). Nous avons pour cela mené une analyse du discours (Giglione et Matalon, 1978) en centrant sur la connotation des propos ;

- la densité (rapport entre les liens effectifs et le total des liens possibles entre les actants). La densité se mesure sur la base de la nature des liens. Ainsi plus les acteurs du réseau apportent de nombreuses ressources, plus le réseau ressources sera dense. L'expérimentation bénéficiera alors d'un environnement plus favorable.

Considérons d'abord ce qui s'est produit en 2007-2008 pour ce qui concerne les ressources. Nous comptabilisons comme ressources : l'engagement, la reconnaissance, la satisfaction, l'attribution d'heures ${ }^{22}$, dont nous avons trouvé les traces dans les entretiens et carnets, et nous faisons l'hypothèse de la réflexivité (un acteur est en général suffisamment narcissique pour s'apporter à lui-même des ressources). Si nous récapitulons les apports de ressources dans une matrice (en annexe), nous pouvons repérer 24 actants engagés dans l'expérimentation : l'IPR, le proviseur et son adjoint, 5 enseignants, 14 élèves, les objets : les systèmes, considérés comme un seul actant et enfin l'espace. L'étendue du réseau ressources est donc de 24.

Nous avons vu plus haut que si certains acteurs apportaient des ressources, d'autres au contraire dressaient des obstacles. Nous avons donc qualifié les liens (r pour ressources, o pour obstacle) dans la matrice qui se lit en ligne en respectant scrupuleusement les propos des acteurs et leur connotation. 
Enfin, nous avons calculé la densité du réseau ressources et celle du réseau obstacles. Pour ce qui concerne le réseau ressources : Densité exprimée en pourcentage $=$ [nombre de liens apportant des ressources]/[nombre total maximal de liens apportant des ressources]. Le nombre maximal de $100 \%$ ne peut être atteint que si tous les actants apportent des ressources à tous les autres et à eux-mêmes, ce qui est fort improbable. Pendant l'année 2007-2008 la densité du réseau ressources est donc :

$$
69 /\left(24^{2}\right)=69 / 576=11,9 \%
$$

51 En procédant de la même façon pour les obstacles (matrice en annexe), nous obtenons pour le réseau obstacles une densité de $7,1 \%$. Si nous faisons le même exercice pour l'année suivante, nous pouvons comparer les résultats.

Comparaison des étendues et des densités des Réseaux ressources et obstacles en 2007-2008 et 2008-2009

\begin{tabular}{|l|l|l|l|l|}
\hline & \multicolumn{2}{|l|}{ Année 2007-2008 } & \multicolumn{2}{l|}{ Année 2008-2009 } \\
\hline & étendue & Densité & étendue & Densité \\
\hline Réseau ressources & 24 & $11,9 \%$ & 26 & $14,6 \%$ \\
\hline Réseau obstacles & 24 & $7,1 \%$ & 26 & $6,65 \%$ \\
\hline
\end{tabular}

D'une part, l'étendue du réseau a cru. En effet, Sciences + s'est ouvert au club d'astronomie et notre présence a suscité l'intérêt de l'inspection. D'autre part, la densité du réseau ressources a augmenté avec le temps (de 11,9\% à 14,6\%). Le réseau est donc plus riche en ressources. Alors que la densité du réseau obstacles décroît (de $7,11 \%$ à $6,65 \%$ ), le réseau est moins porteur d'obstacles.

53 Notons que la densité du réseau ressources est toujours supérieure à celle du réseau obstacles; c'est lié exclusivement à notre choix de la réflexivité en matière de ressources.

54 Les mesures du réseau sont donc susceptibles d'apporter un éclairage complémentaire à celui de l'ANT, en objectivant le changement. En effet, si les acteurs avaient l'intuition d'une évolution favorable de leur expérimentation, ils n'en avaient aucun signe objectif. L'étendue et la densité du réseau ressources (présentes dans les discours et les représentations des acteurs) sont un levier favorable à une expérimentation, en parallèle bien entendu avec la décrue des obstacles.

\section{Conclusion}

Cette expérimentation, placée sous l'égide de l'article 34, connaît de nombreux problèmes d'installation bien qu'il semble, au regard de l'analyse que nous avons présentée ici, qu'elle ait progressé, ce que confirme d'ailleurs le bilan intuitif de ses promoteurs, de la direction de l'établissement dans lequel elle est menée et du corps d'inspection, bien qu'il ne l'ait pas encore visitée.

L'extension du réseau, que nous avons mesuré par l'étendue est un des points de passage obligés (PPO) de cette progression. En effet, de nombreuses études (recensées par exemple dans Lessard, Kamanzi et Larochelle, 2009) ont montré que le travail d'une équipe était plus efficient que celui d'un enseignant solitaire. Finalement le réseau agit 
ici comme pour le lien social : les personnes qui ont un nombre important de relations sont les mieux intégrées dans la société, contrairement aux « exclus ${ }^{23}$ " qui ont peu de relations (Paugam, 2007), un réseau étendu est plus protecteur. Il en est de même dans un établissement scolaire ou dans une équipe unie autour d'un projet. Plus les partenaires sont nombreux, à condition d'être enrôlés c'est-à-dire apportant des ressources et posant moins d'obstacles, plus le réseau est intégrateur et permet un laboratoire, au sens de Latour, plus actif. En effet chaque membre du réseau est susceptible d'alimenter le débat et de nourrir ainsi la problématisation de l'activité.

L'enrôlement des acteurs, qui pouvait a priori être perçu comme intuitif prend plus de sens lorsqu'on le mesure dans les propos des acteurs, en mettant l'accent sur les ressources véhiculées par les liens du réseau des actants. Ces ressources étant soit symboliques (reconnaissance), soit matérielles (rémunération, temps, lieu d'exercice). La présence de ressources en quantité et en qualité suffisantes est aussi un des PPO. Ce sont ces ressources qui font tenir la chaîne relationnelle et empêchent des bifurcations qui se feraient aux dépens de l'innovation.

Enfin, la mesure de la densité permet de lier étendue du réseau et nature de l'enrôlement et surtout de donner une mesure objective du réseau constitué autour de l'expérimentation. Bien sûr il serait souhaitable, la recherche est encore en cours, d'affiner notre instrument, en valuant les ressources, ce qui permettrait de calculer un indicateur unique de densité (avec des ressources notées positivement et des obstacles négativement). Néanmoins, nous nous heurtons ici à la complexité du social. Considérer les faits sociaux comme des choses, pour paraphraser Émile Durkheim (1894), n'est pas aisé, surtout quand le sociologue adopte la démarche forcément compréhensive d'une méthodologie fondée sur des entretiens et cahiers.

De la même manière, et avec la même réserve, lorsque l'expérimentation ne trouve pas ces points de passage, ce qui est arrivé la première année surtout, et ne peut procéder à sa propre remédiation par essais/erreurs, elle s'égare. Son chemin passant par un réseau lâche autorise des objectifs confus, empêche la constitution de ressources, nourrit l'absence de convergence des logiques des acteurs ce qui engendre la méfiance sinon l'hostilité des acteurs entre eux, et in fine empêche la réussite de l'expérimentation. Là aussi, l'analyse en termes de réseaux sociaux trouve sa pertinence. L'étendue du réseau s'avère étroite, l'équipe constituée subit ou choisit l'autarcie. En ce qui concerne les liens du réseau, s'ils peuvent véhiculer des ressources, ils peuvent aussi transporter des obstacles, que l'on peut évaluer en calculant la densité du réseau.

Si la notion de réseau est aussi utilisée pour désigner les infrastructures comme celle de l'électricité, du gaz, ou encore du réseau ferroviaire, il ne faut pas oublier que ce qui compte dans un réseau c'est ce qu'il transporte. Ce que résume le sociologue des réseaux sociaux Harrison White (2008), "no matter the pipes, the important is what's in the pipes ». 


\section{BIBLIOGRAPHIE}

Blais, M.-C., Gaucher, M. \& Ottavi, D., Conditions de l'éducation, Paris, Stock, 2008.

Callon, M., Éléments pour une sociologie de la traduction. La domestication des coquilles saintjacques et des marins pêcheurs dans la baie de Saint-Brieuc, L'année sociologique, $n^{\circ} 36,1986$, p. 57-63.

Charpak, G., Léna, P. \& Quéré, Y., L'Enfant et la science. L'aventure de la main à la pâte, Paris, Odile Jacob, 2005.

Convert, B., Les Impasses de la démocratisation scolaire : Sur une prétendue crise des vocations scientifiques, Paris, Raisons d'Agir, 2006.

Cros, F., L'Innovation à l'école : forces et illusions, Paris, PUF, 1993.

Cros, F. (dir.), Dynamique du changement en éducation et formation, Paris, INRP, 1998.

Cros, F. (dir.), Dynamique du changement en éducation et formation, Paris, INRP, 2001.

Cros, F. (a), « Emergence et installation de l'innovation scolaire : pertinence de la théorie de la traduction ", Bronckart J.-P. et Gather-Thurler (Eds), Transformer l'école, Bruxelles, De Boeck. 2004 a, p. 59-78.

Cros, F. (b), L'Innovation scolaire aux risques de son évaluation, Paris, L'Harmattan, 2004.

Degenne, A. \& Forsé, M ., Les réseaux sociaux. Une approche structurale en sociologie, Paris, Armand Colin, 1994.

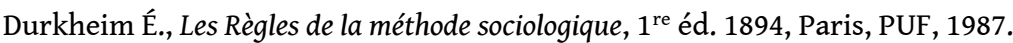

Eurydice, L'Enseignement des sciences dans les établissements scolaires en Europe. État des lieux des politiques et de la recherche, Bruxelles, 2006.

Fourez G., La Construction des sciences. Les logiques des inventions scientifiques, Bruxelles, De Boeck, 2002.

http://books.google.fr/books?id=ou1BwfxzqIkC\&printsec=frontcover\&dq=Gérard + Fourez + sciences $\&$ source $=$ bl \& ots $=$ AVHNcXD3s7 \& sig =-F1ArwtMsPjApY-481IIVWiL_RE \& hl $=$ fr \& ei $=$ h_42TOarAoOIOKnKqOYK $\&$ sa $=$ X \& oi $=$ book_result $\& \mathrm{ct}=$ result $\&$ resnum $=2 \&$ ved $=0 C B k Q 6 A E W A Q \# \mathrm{v}=$ onepage \& $\mathrm{q} \& \mathrm{f}=$ false (disponibilité partielle, le 9 juillet 2010).

Gadéa, C., « Sociologie des sources, sociologie des fontaines ", Cros, F. (dir.), Dynamiques du changement en éducation et en formation. Considérations plurielles sur l'innovation, Paris, INRP, 1998, p. 21-33

Gather Thurler, M., «Innovation et coopération entre enseignants : liens et limites », Bonami, M. \& Garant, M. (dir.), Systèmes scolaires et pilotage de l'innovation. Émergence et implantation $d u$ changement, Bruxelles, De Boeck, 1996, p. 145-168.

Giglione, R. \& Matalon, B., Les enquêtes sociologiques : théories et pratiques, Paris, Armand Colin, 1978.

Giust-Desprairies, F., « Problématique identitaire d'un collectif innovant », Cros, F. (dir.), Dynamiques du changement en éducation et en formation. Considérations plurielles sur l'innovation. Paris : INRP, 1998, p. 49-72. 
Grossetti, M., " Une méthode narrative quantifiée d'analyse des mobilisations de relations ", Actes de la journée d'étude du RT 26 de l'AFS : Nouvelles approches, nouvelles techniques en analyse des réseaux sociaux, Villeneuve d'Ascq, USTL-Clersé., 2008.

Havelock, R.G. \& Huberman, A.M., Innovation et problèmes de l'éducation. Paris : Unesco/BIE, 1980.

Huberman, A.M., « Comment s'opèrent les changements en éducation : contribution à l'étude de l'innovation », Expériences et innovations en éducation, n 4, Paris, Unesco/BIE, 1973.

Latour, B., Les Microbes : guerre et paix des microbes suivi de Irréductions, Paris, Métailié, 1984.

Latour, B., Changer de société, refaire de la sociologie, Paris, La Découverte, 2007.

Lebeaume, J., L'Enseignement des sciences à l'école : des leçons de choses à la technologie, Paris, Delagrave, 2008.

Lessard C., Kamanzi P.C. \& Larochelle M., De quelques facteurs facilitant l'intensification de la collaboration au travail parmi les enseignants : le cas des enseignants canadiens, Éducation et sociétés, 23-1, 2009, p. 59-78.

Mitchell, J.C., Numerical Techniques in Social Anthropology, Philadelphia, Ed. Institute for the Study of Human Issues, 1980.

Musset, M., Sciences en classe, sciences en société, Paris, INRP, 2009 sur :

http://www.inrp.fr/vst/LettreVST/45-mai-2009.htm. Consulté en décembre 2009.

Obin, J.-P., « Les politiques publiques d'innovation scolaire en France depuis 1968 », Cros, F. (dir.), Dynamiques du changement en éducation et en formation. Considérations plurielles sur l'innovation, Paris, INRP, 1998, p. 329-345.

OCDE, Évolution de l'intérêt des jeunes pour les études scientifiques et technologiques : rapport d'orientation, 2006 sur :

http://www.oecd.org/dataoecd/60/24/37038273.pdf. Consulté en décembre 2009.

Paugam S. (dir.), Repenser la solidarité. L'apport des sciences sociales, Paris, PUF, 2007.

Prost, A., Les lycées et leurs études au seuil du XXI siècle, Rapport du groupe de travail national sur les seconds cycles au directeur des lycées, 1983.

Rocard, M. (prés.), L'enseignement scientifique aujourd'hui : une pédagogie renouvelée pour l'avenir de l'Europe, Bruxelles, Commission Européenne, 2007, http://ec.europa.eu/research/sciencesociety/document_library/pdf_06/report-rocard-on-science-education_fr.pdf. Consulté en décembre 2009.

Wittorski, R., « Production d'innovations, développement des compétences et évolution des professionnalités enseignantes », Cros, F. (dir.), Dynamiques du changement en éducation et en formation. Considérations plurielles sur l'innovation, Paris, INRP, 1998, p. 119-143.

White H., Réseaux et récits. Conférence inaugurale à l'école thématique CNRS « Réseaux sociaux : enjeux, méthodes, perspectives ». Cargèse. Septembre 2008.

\section{NOTES}

1. Le caractère dérogatoire a été retenu dans l'académie de Nantes pour différencier innovation et expérimentation.

2. Pour une riche bibliographie de ces monographies, voir Huberman, 1973. 
3. L'expression figure dans le titre de l'ouvrage : L'enfant et la science. L'aventure de la main à la pâte.

4. Soulignons que le sociologue bourdieusien Bernard Convert (2006) ne partage pas cette thèse, pas plus que le didacticien des sciences Gérard Fourez (2002), néanmoins il pense qu' « on enseigne souvent les sciences d'une façon si dogmatique qu'elle rappelle parfois l'enseignement de la religion il y a quelques siècles » (Fourez, 2002, p. 51) et en appelle à l'interdisciplinarité.

5. Les journées d'étude S-Team en octobre 2009 à Grenoble (textes à paraître) étaient centrées sur le collège, celles de l'INRP, en février et mars 2009 à Nantes, en novembre 2009 à Lyon, cette dernière était centrée sur le lycée. Enregistrements disponibles sur http://www.inrp.fr/formation-formateurs.

6. Latour (2007) utilise l'acronyme ANT (Actor Network Theory) pour désigner son paradigme.

7. «Si un acteur n'introduit aucune différence ce n'est pas un acteur.» (Latour, 2007, p. 190.)

8. L'ANT est considérée comme une branche de l'analyse des réseaux aux côtés des branches de l'analyse sociométrique, de l'analyse structurale des réseaux, de l'anthropologie structuro-fonctionnaliste britannique, de l'analyse configurationnelle et celle des chaînes. Pour une présentation synthétique : Degenne \& Forsé (1994).

9. Cette recherche est encore en cours. Nous avons enquêté dans 4 des 8 établissements engagés dans une expérimentation article 34 dans l'académie de Nantes où nous avons enquêté. Cette recherche est menée sous la direction d'Yves Dutercq. Elle est soutenue par le rectorat de Nantes et l'INRP (UMR Educpol).

10. Les expérimentations sont soumises à évaluation tous les ans. Elles peuvent garder le statut d'expérimentation pendant cinq ans.

11. Il ne s'agit pas du paradigme de l'ANT.

12. Un IPR de technologie a été contacté mais a été dans l'impossibilité de nous recevoir.

13. Le carnet offre par conséquent un complément d'information.

14. La MIVIP (mission de valorisation des innovations pédagogiques) est une mission académique du rectorat de Nantes qui «a pour rôle de repérer, d'encourager, de valoriser et de diffuser les initiatives locales réalisées dans le primaire et le secondaire ».

15. Comme la coquille Saint-Jacques qui refusait de se reproduire dans l'article fondateur de la sociologie de la traduction de Michel Callon (1986).

16. Les mouvements des planètes sont au programme de seconde.

17. Nous sommes conscientes de l'ambiguïté de la commande, mais ne nous interrogerons pas ici sur ce sujet.

18. Dans une lunette la lumière est déviée par un miroir alors que sur un télescope elle ne l'est pas.

19. Inspection pédagogique régionale, Synthèse Roberval, octobre 2009.

20. Un des fondateurs de la psychologie sociale, Elton Mayo (1927) a expérimenté, dans les ateliers Hawthorne à la Western Electric, le rôle de l'éclairage dans l'évolution de la productivité des travailleurs. Or, le simple intérêt du chercheur la provoquait, puisque 
la non modification de l'éclairage dans l'atelier placébo s'accompagnait tout de même d'une amélioration de la productivité de ses ouvriers.

21. Ce qui est le cas ici: c'est le réseau de l'ensemble des acteurs concernés par l'expérimentation sachant qu'« aucun réseau ne possède de frontière naturelle. C'est le sociologue qui fixe les frontières ». (Degenne \& Forsé, 1994, p. 30).

22. Les ressources qui pourraient être apportées en dehors de l'expérimentation ne sont pas comptabilisées car elles n'appartiennent pas au réseau complet de l'expérimentation.

23. Le terme d'exclu n'est pas un concept sociologique. Nous ne l'employons que par commodité...

\section{RÉSUMÉS}

Les expérimentations conjuguant Sciences Expérimentales et Sciences de l'Ingénieur en classe de seconde (article 34 de la loi d'orientation de 2005) s'appuient pour leur mise en place et pour se pérenniser sur des ressources véhiculées par des réseaux sociaux: aide didactique de l'inspecteur, heures rémunérées... Mais, ces expérimentations se heurtent également à des obstacles: absence d'enrôlement, de reconnaissance, problèmes spatiaux et techniques. En présentant une étude de cas, menée lors de deux années consécutives, nous verrons comment l'enrôlement des acteurs, un laboratoire plus actif ainsi que l'extension et l'accroissement de la densité du réseau sont les points de passage obligés du chemin d'installation.

For their installation and also to perpetuate, experimentations in Experimental Sciences and Engineering (article 34 of the law of orientation of 2005) in the first year of high school lean on resources conveyed by social networks : the didactic help of the inspector, paid hours... But they also collide with obstacles : lack of recognition, of motivation of the pupils, space or technical problems. By leaning on a case, studied during two successive years, we'll see how the enlistment of the actors, a more active laboratory as well as the expansion and density growth of the network are the obligatory passages points of the roads of installation.

\section{AUTEURS}

\section{XAVIÈRE LANÉELLE}

(IUFM des Pays de-la-Loire-Université de Nantes, CREN)

\section{ISABELLE HARLÉ}

(IUFM des Pays de-la-Loire-Université de Nantes, CREN) 